\title{
Management of deep pectus excavatum (DPE)
}

\author{
Jose Ribas Milanez de Campos, Miguel Lia Tedde \\ Hospital das Clinicas, University of São Paulo, Hospital Israelita Albert Einstein, São Paulo, Brazil \\ Correspondence to: Jose Ribas Milanez de Campos. Hospital das Clinicas, University of São Paulo, Hospital Israelita Albert Einstein, São Paulo, Brazil. \\ Email: jribas@usp.br.
}

\begin{abstract}
The correction of deep pectus excavatum, with the Nuss procedure, frequently require a series of maneuvers that is inherently dangerous. Herein we describe 10 technical modifications to prevent potential complications. These modified techniques have certain advantages and according to the authors, with these maneuvers the risk of pericardial sac, cardiac injury, bar displacement and complications during the removal of the bar could be markedly reduced.
\end{abstract}

Keywords: Pectus excavatum; Nuss procedure; crane technique; anthropometric index

Submitted Apr 06, 2016. Accepted for publication Aug 15, 2016.

doi: $10.21037 /$ acs.2016.09.02

View this article at: http://dx.doi.org/10.21037/acs.2016.09.02

\section{Introduction}

To the best of our knowledge, the medical literature does not contain an established definition for deep pectus excavatum (DPE), although pectus excavatum (PE) itself comprises approximately almost $90 \%$ of all chest wall deformities and is mainly corrected through surgery.

For years, open surgery techniques were the golden standard for treating PE. However, this situation changed when a minimally invasive repair technique (MIRPE) was introduced by Donald Nuss (1). The MIRPE procedure requires the creation of a substernal tunnel for the positioning of a bar that helps restore proper chest architecture. The advancement of the introducer between the sternum and the heart is a dangerous maneuver and is the riskiest step of the procedure. In some patients, as described in medical literature, cardiac perforation has occurred during creation of the retrosternal tunnel (2).

Severe, asymmetrical, and DPE deformities are not rare, and the decision for repair is based on the severity of symptoms, deformity based on the pectus index, physiologic and quality of life factors. Patients who do not undergo repair during childhood often experience progressive worsening of symptoms during adulthood. To this date, there are few published reports regarding pectus repair in such patients. The purpose of this article is to describe a series of maneuvers and technical modifications that avoid not only pericardial and cardiac injury, the most dramatic of the possible complications of the procedure, but also dislocation of the bar and injury to the intercostal muscles produced by the enormous pressure of the bar over the ribs when performing MIRPE, particularly in these cases.

\section{Methods}

The literature reports a series of technical modifications made to the original MIRPE technique. Among those alterations are some that were specifically conceived to prevent problems at the riskiest stage of the procedure, which is the creation of the retrosternal tunnel. Our group has contributed to some of those modifications $(3,4)$, and has added steps to comprise what is our current MIRPE technique for difficult cases.

Although most ordinary cases do not require the adoption of the maneuvers herein, we believe it is important for any surgeon planning to perform corrective PE to have those techniques in mind as an armamentarium that will be most valuable in DPE cases.

\section{Clinical parameter with anthropometric index (AI)}

The need to objectively assess patients with DPE and compare the results of several treatment techniques that 
can readily be reproduced in ambulatory practice at low cost motivated us to develop the Anthropometric Index (AI). The AI is defined as the ratio between the maximum depressions across the maximum external sagittal diameter at the deepest point of the deformity, or at the distal third of the sternum (5).

The measurements are taken with the patient in the horizontal supine position on a flat table during deep inspiration and with the aid of simple instruments. The comparison of these measurements with the Haller Index (HI), which has been adopted by most centers, showed that there was significant correlation between both indexes, and similar accuracy (6). The AI has additional advantages of avoiding radiation exposure for the patient and can be quickly determined in ambulatory practice at a very affordable cost (7) (Figure 1).

\section{Position of the patient on the surgical table}

Since 2006, we have proposed a new position on the surgical table, wherein the patient is placed in the supine position longitudinally on the surgical table (Figure 2), on a $12 \mathrm{~cm}$-high cushion that extends from the back of the head to the waist, so that the chest protrudes upwards with the arm alongside the body below the level of the incisions and fixed to the surgical table. The optical trocar in this position is introduced in a more cranial orientation, followed by the introduction point for the metal bar.

The benefits of this new approach are detailed below:

(I) Increased safety during the introduction of the trocar, as there is reduced risk of diaphragmatic injury;

(II) Easier handling of the optical device enables better visualization of the pleural cavity and bar inserter during dissection of the anterior mediastinum;

(III) Better positioning of a thoracic drain, if necessary;

(IV) An aesthetically more acceptable result, with surgical scars covered by the patient's arms;

(V) Prevents brachial plexus injury.

\section{Non-invasive "Crane" technique}

The literature reports several different maneuvers that aim to elevate the sternum during the opening of retrosternal tunnel. Although most of these maneuvers seem to be quite effective, their main drawback is that they are based on the use of dedicated instruments that are usually unavailable. With this in mind, we developed maneuvers
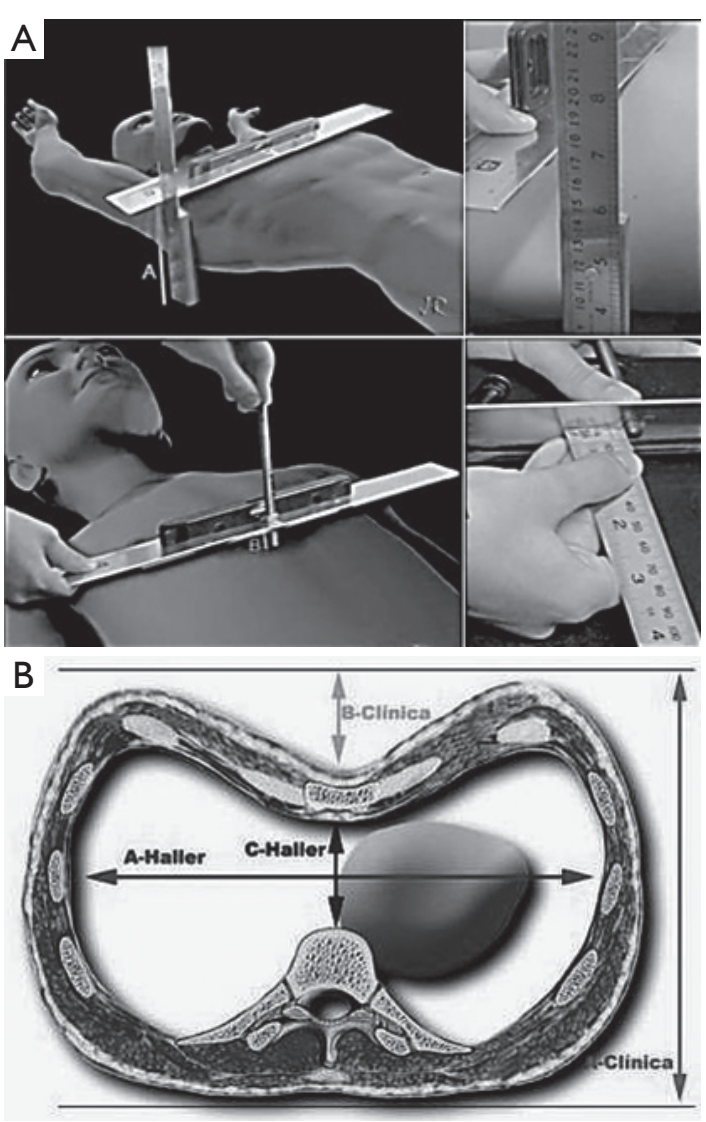

Figure 1 Anthropometric index = measurement of distance (A) and (B).

based on ordinary surgical instruments that are present in any thoracic instrumental surgical kit. The technique is described elsewhere (8), but a short description follows:

(I) The skin is marked at the point of deepest depression along the midline and where the ideal hinge points in each hemithorax should be placed. A subcutaneous tunnel is created from the skin incisions in the direction of the hinge points. We first enter the left hemithorax. This maneuver allows us to perform digital exploration and position a Langenbech handheld retractor inside the left thoracic cavity. In the right hemithorax, the camera is introduced and a second Langenbech retractor is positioned at the hinge point. Both retractors are then pulled upwards, bringing the sternum along with them as the heart sinks downwards under its own weight. The gap that is created allows for safe retrosternal instrumentation. Having transversed the substernal space with the introducer, the left Langenbech retractor can be 


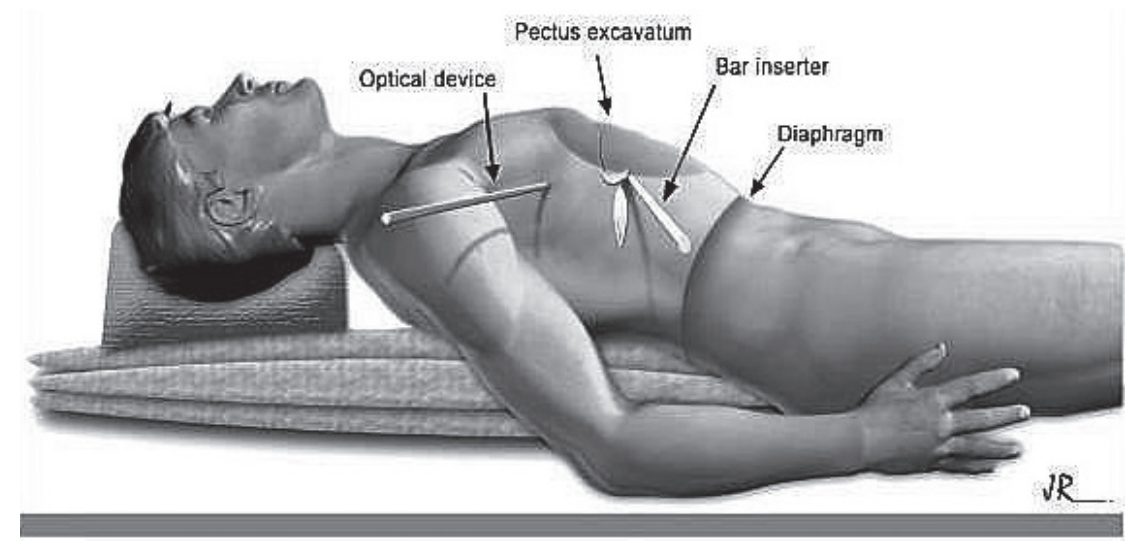

Figure 2 Surgical position.
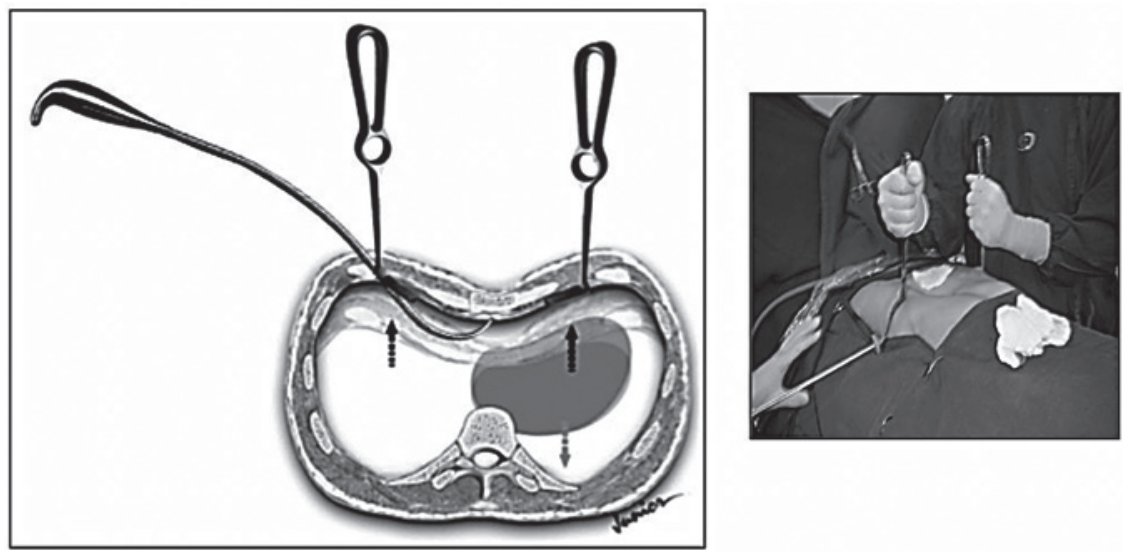

Figure 3 The Langenbeck retractor lifts the chest wall.

removed with the tactile aid of the surgeon's finger, helping to guide the introducer out of the thoracic cavity in a safe way (Figure 3);

(II) The Vacuum Bell device, without its hand pump and directly connected to a wall vacuum source, is used intraoperatively during the MIRPE procedure. Suction on the anterior chest wall expands the retrosternal space, ensuring safe passage of the introducer (Figure 4). Special care was exercised whenever the patient was not a regular user of the Vacuum Bell. In these cases, the device was not kept over the chest wall for prolonged periods in order to avoid formation of subcutaneous hematomas (9);

(III) The Crane technique, by Park et al. (10) recommended elevating the depressed sternum prior to the insertion of the introducer, by means of wire sutures threaded through the sternum to a retracting system mounted over the operating table. The crane offers two main advantages: in adults, it alleviates the pressure on the bar and at the hinge points due to their relatively heavy and stiff chests. It also prevents internal organ injury during the passage of the introducer (Figure 5);

(IV) The Dawn Jaroszewski technique (11), described as a forced mechanical elevation of the sternum which increases the retrosternal anterior-posterior dimensions. Its advantages include increased thoracoscopic visualization of the dissection area, minimizing potential trauma to the aorta, pericardium and heart, as well as reduced risk of intercostal muscle stripping in adult patients undergoing MIRPE using a bone clamp and Rultract retractor (Figure 6);

(V) The Johnson et al. technique (12) describes the use of a scope in the right hemithorax as a subxiphoid incision is made. The xiphoid is removed and a plane 


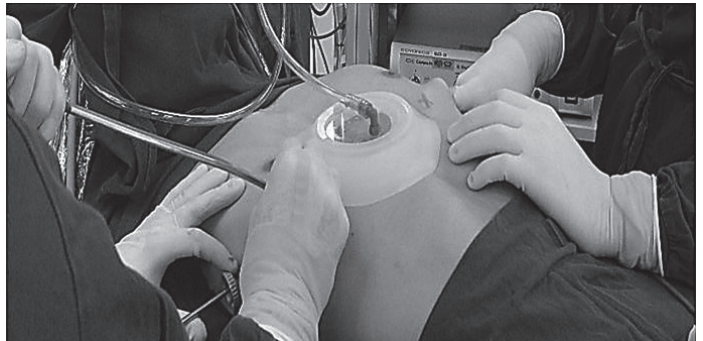

Figure 4 Vacuum bell intraoperatively.

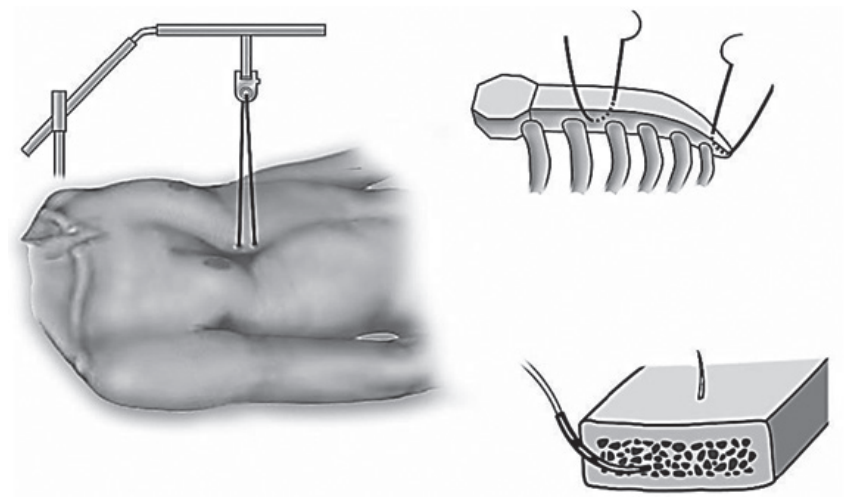

Figure 5 Steps for the Park Crane technique.

between the pericardium and posterior sternum is created using digital dissection. Using the finger as a tactile guide, a lift is inserted beneath the sternum, creating a gap between the pericardium and sternum which improves visibility and safety.

Objective targets to create a substernal tunnel: starting on the left side

Arrows indicate the marks on the skin under which the subcutaneous tunnel is created and the entrance incision in the left hemithorax is made first. In the opposite direction, in the right hemithorax, there is the exit incision, or hinge point, which is the 'target' (Figure 7). The tip of the introducer is inserted through the entrance incision and from right to left, is gradually threaded towards the exit point in the right hemithorax. The tip remains in plain sight all along the subcutaneous segment under thoracoscopic visualization, which also allows for minimal rubbing of the device against the pericardium. The exit path can also be guided by palpation, as the surgeon's finger is already inserted in the left incision, ready to receive the oncoming

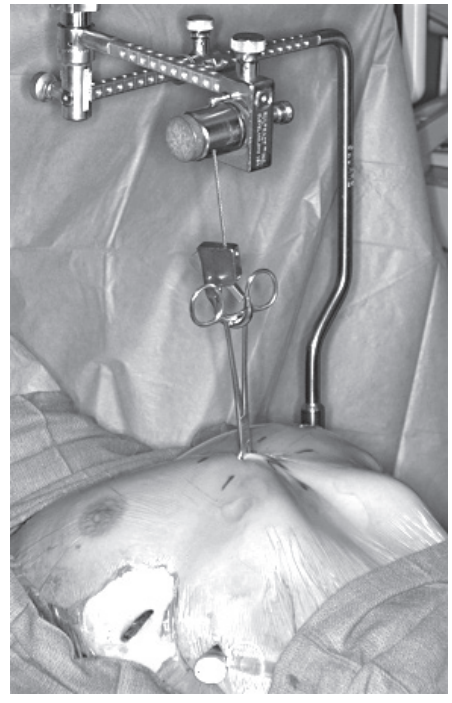

Figure 6 Sternal elevation during MIRPE.



Figure 7 Position of the skin marks and starting from the left side.

tip of the introducer.

The creation of the retrosternal tunnel runs more smoothly when you are guided to these 'objective targets', and we can literally say that there is 'light at the end of the tunnel'. Since we started using this technique, we have not experienced any technical difficulties when creating the substernal tunnel (8).

\section{Shorter bars and two stabilizers in a more medial position}

Bar displacement is a serious complication that can occur anytime, but most frequently within the 30-day period following MIRPE. Several modifications have been proposed in an effort to make the technique safer and more effective, including the use of shorter bars and new positioning of the stabilizers, avoiding pericostal sutures or other methods. In one group of 51 patients after 2008, De 

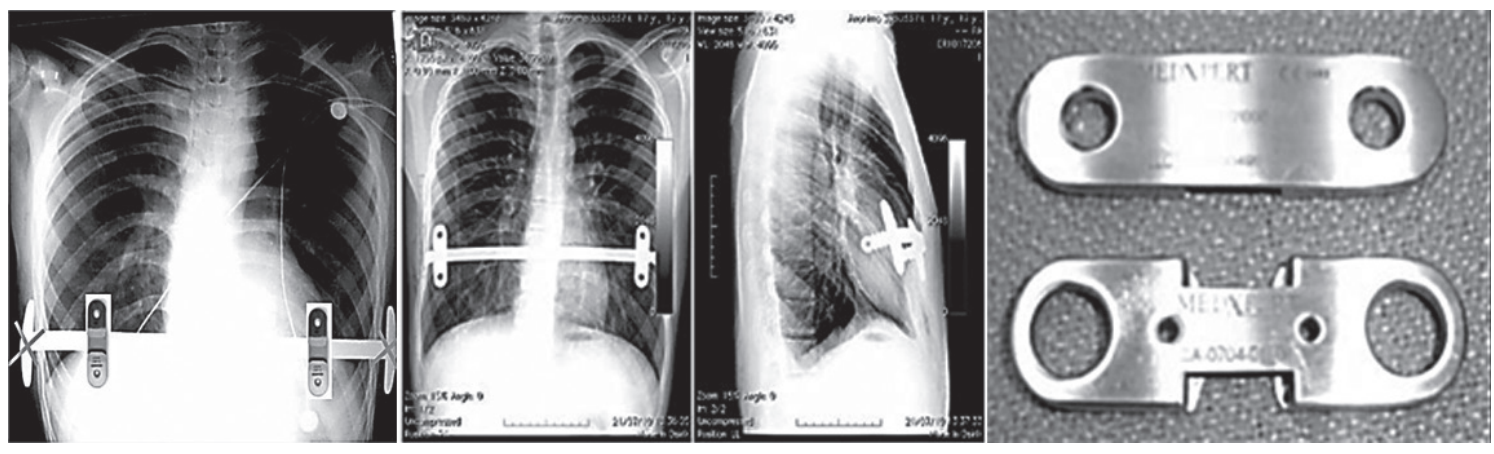

Figure 8 One stabilizer is placed on each side of the bar as close as possible to the hinge point to avoid bar displacement.

Campos et al. (13) we also abided by the recommendations set forth by Pilegaard et al. (14) and performed MIRPE with two technical modifications, including a smaller bar size and the use of a newly designed stabilizer model. This new model, with central grooves on the posterior surface, allows for improved sliding of the stabilizer over the bar, regardless of its curvature. This property ensures a more medial positioning of the stabilizer (Figure 8). We elected to use one stabilizer at either side of the bar, distributing the forces to at least two ribs. We would like to add that, according to Watanabe et al. (15), due to its size and when in the lateral position, the stabilizers increase the incidence of wound complications such as seroma and dermatitis due to pressure damage, or even skin erosion. When the stabilizer was placed medially, it was at least partially covered by the pectoralis major muscle.

\section{One, two, three or more bars?}

At present, two bars are increasingly used and the complication rate has not increased. In fact, better cosmetic results and less pain were observed in many cases where two bars were inserted, sometimes through different or the same incisions. Double bar positioning can be adopted if the excavation is deep and short. The two bars may be crossed to provide the best support under the deepest point of the PE. In almost all cases, the superior or high bars should be inserted first in order to elevate the sternum and consequently allow for safer and easier placement of the second bar as also proposed by Pilegaard et al. in several papers $(14,16)$.

According to the same author (16), since 2011, the use of more than one bar was significantly common in patients over 30 years of age. In such older patients, two stabilizers were used in $10 \%$ of cases, instead of the traditional use of only one stabilizer on the left side. In our practice, we use two stabilizers on each bar, and sometimes four stabilizers in two bars. More importantly, we attach the stabilizers to each other using steel stiches in order to build a bridge linking both bars for better stability (Figure 9).

Grosen et al. (17) have shown that older patients may require more morphine than younger patients, a fact that may be explained by age-related rigidity of the thoracic cage. Even with this, more rigid chest wall, we have been able to correct all patients with good results and with pain control, allowing for discharge at the same time as younger patients. Another explanation for these outcomes may be our frequent use of more than one bar. Nagaso et al. (18) has also shown that patients with two bars experience less pain than patients with only one. The theoretical explanation could be that more bars deliver the required force to the ribs over a larger surface, making the force per unit of area less intense.

\section{Release the cartilage tension}

In most of the DPE cases herein, the occurrence of calcified ribs, a fixed thoracic cage, overlapping redundant cartilages, and mixed PE deformities was frequent. During the past 13 years, we have resected only short segments of costal cartilages. Our technique includes a $5 \mathrm{~cm}$ longitudinal incision made over a short midline, depending on the number of deformed cartilages. A subperichondrial resection of short segments adjacent to the sternum near or beyond the costochondral junction was often made. Sometimes we needed a transverse wedge osteotomy across the anterior table of the sternum. The posterior table of the sternum is gently fractured and the lower sternum elevated and twisted, when asymmetric, to the desired level. Subsequently, both pleural spaces were opened and 

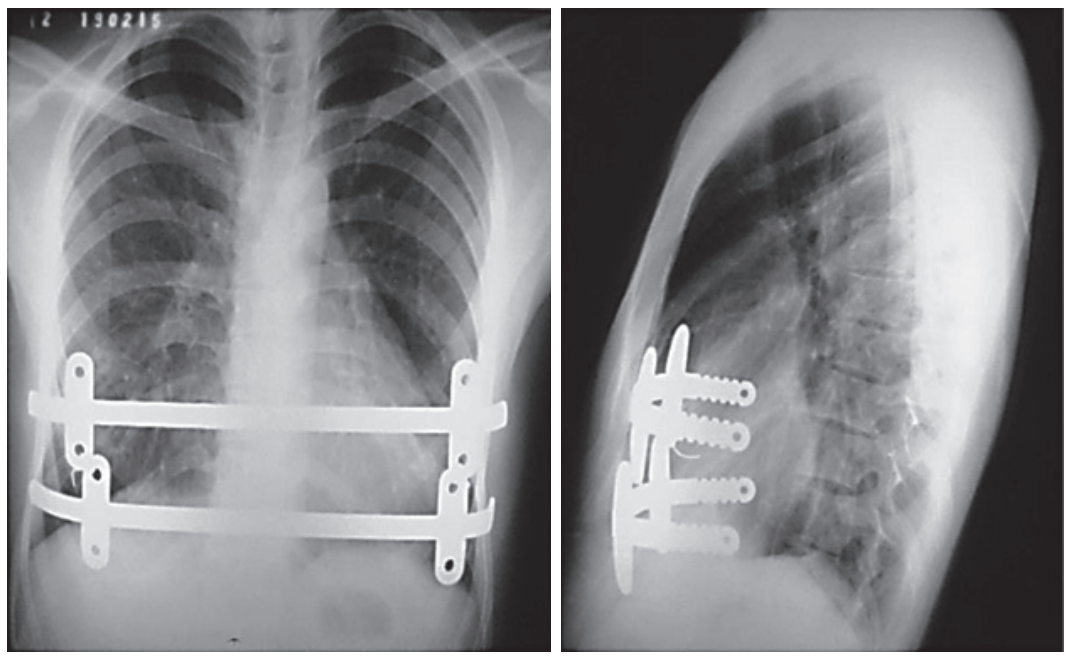

Figure 9 Two bars and four stabilizers as a bridge linking the bars.

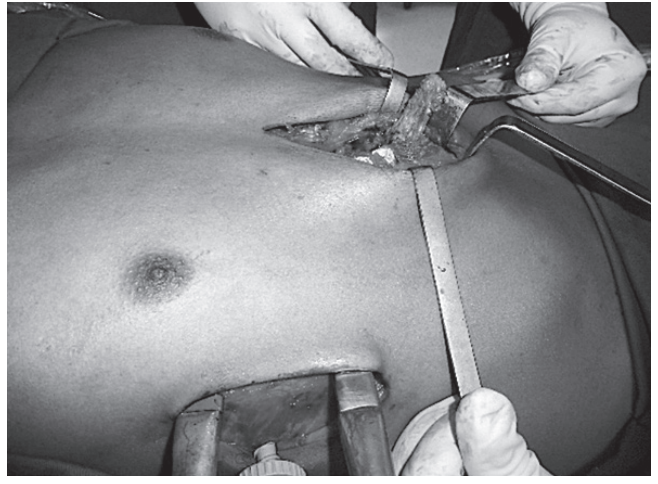

Figure 10 A longitudinal incision made over a short midline to remove short segments of costal cartilages.

a support strut was placed posterior to the sternum. We had the opportunity to use different models of bars but, after 2003, we have used the same kind of bar we use to perform MIRPE. The costal cartilages were shortened, if possible, with interrupted stiches. The pectoralis and abdominal muscles were reconstructed to completely cover the cartilaginous repair. Sometimes, we also used a mesh to finish this maneuver. A hemovac drain was placed between the cartilage and muscle repairs. The subcutaneous tissues and skin were closed with interrupted and absorbable sutures. As mentioned before, all DPE patients as well as those with recurrent deformities had a support strut with one or two stabilizers to hold the bar in place (Figure 10).

We would like to mention the another technique proposed by Varela et al. (19) where the cartilages are cut thoracoscopically from the inside to decrease the rigidity of the chest. Although minimally invasive repair of pectus has gained worldwide acceptance, a thoracoscopic approach with multiple cartilage incisions for all anomalous costal cartilages has been rarely done. The cartilages can be removed progressively using a rongeur, preserving the anterior perichondrium. The authors initially proposed this technique as a feasible alternative for the treatment of unilateral pectus carinatum, but it can also be used for bilateral treatment of DPE with the same purpose of releasing the cartilage tension.

\section{Combination approach for complex repair}

Repair of DPE using MIRPE combined with the 'open technique' for minimal cartilage resection is slightly more technically difficult than MIRPE alone, because the costal cartilages in adults are often rigid, deformed, and partially ossified. However, this technique produces good results more consistently, with lower morbidity, less pain, shorter hospitalization and more rapid return to full activity, as also mentioned by Jaroszewski and Fonkalsrud (20).

Resection of all of the deformed costal cartilages, as in the Ravitch repair, is unnecessary and may damage the periosteum and the cartilage growth centers, often resulting in ossified rigid regenerated cartilage that prevents optimal results. Most symptoms caused by DPE can be improved considerably after surgical repair. The technique for repair using MIRPE and minimal cartilage resection with temporary support by an internal strut has provided better 



Figure 11 Combination approach, MIRPE and minimal cartilage resection.

results than when more extensive cartilage resection was adopted (Figure 11).

\section{Small thoracic drain after a complex procedure}

Recent literature suggests that treatment with a small caliber tube is equally effective and less painful than treatment with a large caliber tube for thoracostomy. Because of the risk of a pneumothorax and/or a pleural effusion after a complex thoracic procedure, a chest tube should be considered for all patients with a DPE repair. In our experience, this small thoracic drain (14 Fr) was used for less than 24 hours in cases of pleural effusions and also only for 24 hours after the closure of the air leaks.

\section{Removing the metallic bars with 'notched' ends}

Complications during bar removal were usually related to initial inadequate positioning. During removal, complete alignment of the extremities of the metallic bars is very important to avoid curved bars that may act as a 'hook shape' and cause hemorrhage (13). In our practice, the bars are removed from the side on which the stabilizer was initially fixed following its complete alignment, which was usually the left side. In our experience with 34 cases of bar removal, we observed one case of intra-operative hemorrhage that warranted surgical exploration because of active intercostal bleeding. It is possible that the 'hook shape' of the incompletely rectified bar and sharp silhouette of the bar extremity that passes along the fibrous tunnel may have caused the bleeding. The majority of the bars used worldwide have the notched ends that can be dangerous when this part is trespassing the anterior mediastinum.

We perform maximum bar alignment before removal (Figure 12). In addition, we wrap a rubber film around the distal extremity (notched ends) in order to reduce the risk of bleeding. This very thin film does not increase the thickness of the bar and is closely bound with double wires to avoid the risk of being retained within the tunnel.

\section{Results and final considerations}

Two main reasons prevent us from presenting our results in a numerical and classical fashion: the first is that there is no clear definition of DPE, which makes classification of cases subjective; and second, there is no clear indicator that establishes which of the presented techniques should be used in a given case, which again leads to subjective choices.

In spite of these shortcomings, and based on our experience in PE and DPE, we can safely declare that when adopting the anthropometric index $(21,22)$ for simple measurement and the maneuvers herein, the surgeries were more easily performed and the risk of pericardial sac and cardiac injury was markedly reduced in the treatment of patients who could be classified as DPE, asymmetric deformities, older patients with very stiff thoracic cages, as well as cases in which we have had to use more than one bar or a complex repair rig with the adoption of two or more surgical techniques. In conclusion, until a definitive 



Figure 12 Rubber film protection in a previous aligned bar.

effective and safer MIRPE technique is established, the adoption of the presented techniques in difficult cases enhances safety and outcomes, especially in DPE.

\section{Acknowledgements}

The authors thank Geraldo Avila for assistance with the text and Junior Falcetti for the images.

\section{Footnote}

Conflicts of Interest: The authors have no conflicts of interest to declare.

\section{References}

1. Nuss D, Kelly RE Jr, Croitoru DP, et al. A 10-year review of a minimally invasive technique for the correction of pectus excavatum. J Pediatr Surg 1998;33:545-52.

2. Gips H, Zaitsev K, Hiss J. Cardiac perforation by a pectus bar after surgical correction of pectus excavatum: case report and review of the literature. Pediatr Surg Int 2008;24:617-20.

3. de Campos JR, Fonseca MH, Werebe Ede C, et al. Technical modification of the Nuss operation for the correction of pectus excavatum. Clinics (Sao Paulo) 2006;61:185-6.

4. Tedde ML, Campos JR, Das-Neves-Pereira JC, et al. The search for stability: bar displacement in three series of pectus excavatum patients treated with the Nuss technique. Clinics (Sao Paulo) 2011;66:1743-6.

5. Rebeis EB, Campos JR, Fernandez A, et al. Anthropometric index for Pectus excavatum. Clinics (Sao
Paulo) 2007;62:599-606.

6. Haller JA Jr, Shermeta DW, Tepas JJ, et al. Correction of pectus excavatum without prostheses or splints: objective measurement of severity and management of asymmetrical deformities. Ann Thorac Surg 1978;26:73-9.

7. Brigato RR, Campos JR, Jatene FB, et al. Pectus excavatum: evaluation of Nuss technique by objective methods. Interact Cardiovasc Thorac Surg 2008;7:1084-8.

8. Tedde ML, de Campos JR, Wihlm JM, et al. The Nuss procedure made safer: an effective and simple sternal elevation manoeuvre. Eur J Cardiothorac Surg 2012;42:890-1.

9. Haecker FM, Mayr J. The vacuum bell for treatment of pectus excavatum: an alternative to surgical correction? Eur J Cardiothorac Surg 2006;29:557-61.

10. Park HJ, Jeong JY, Jo WM, et al. Minimally invasive repair of pectus excavatum: a novel morphology-tailored, patient-specific approach. J Thorac Cardiovasc Surg 2010;139:379-86.

11. Jaroszewski DE. Forced mechanical sternal elevation for Nuss repair. Ann Thorac Surg 2013;96:1914.

12. Johnson WR, Fedor D, Singhal S. A novel approach to eliminate cardiac perforation in the nuss procedure. Ann Thorac Surg 2013;95:1109-11.

13. de Campos JR, Das-Neves-Pereira JC, Lopes KM, et al. Technical modifications in stabilisers and in bar removal in the Nuss procedure. Eur J Cardiothorac Surg 2009;36:410-2.

14. Pilegaard HK, Licht PB. Early results following the Nuss operation for pectus excavatum--a single-institution experience of 383 patients. Interact Cardiovasc Thorac Surg 2008;7:54-7.

15. Watanabe A, Watanabe T, Obama T, et al. The use 
of a lateral stabilizer increases the incidence of wound trouble following the Nuss procedure. Ann Thorac Surg 2004;77:296-300.

16. Pilegaard HK. Extending the use of Nuss procedure in patients older than 30 years. Eur J Cardiothorac Surg 2011;40:334-7.

17. Grosen K, Pfeiffer-Jensen M, Pilegaard HK. Postoperative consumption of opioid analgesics following correction of pectus excavatum is influenced by pectus severity: a singlecentre study of 236 patients undergoing minimally invasive correction of pectus excavatum. Eur J Cardiothorac Surg 2010;37:833-9.

18. Nagaso T, Miyamoto J, Kokaji K, et al. Double-bar application decreases postoperative pain after the Nuss procedure. J Thorac Cardiovasc Surg 2010;140:39-44, 44.
19. Varela P, Torre M. Thoracoscopic cartilage resection with partial perichondrium preservation in unilateral pectus carinatum: preliminary results. J Pediatr Surg 2011;46:263-6.

20. Jaroszewski DE, Fonkalsrud EW. Repair of pectus chest deformities in 320 adult patients: 21 year experience. Ann Thorac Surg 2007;84:429-33.

21. Rebeis EB, Samano EM, Fernandez A, Campos JRM, Jatene FB, OliveiraSA. Anthropometric index for qualitative assessment of pectus excavatum. Jornal Brasileiro de Pneumologia 2004;30:501-7. Available online: www.jornaldepneumologia.com.br

22. Rebeis EB, Campos JR, Moreira LF, et al. Variation of the Anthropometric Index for pectus excavatum relative to age, race, and sex. Clinics (Sao Paulo) 2013;68:1215-9.
Cite this article as: de Campos JR, Tedde ML. Management of deep pectus excavatum (DPE). Ann Cardiothorac Surg 2016;5(5):476-484. doi: 10.21037/acs.2016.09.02 